\title{
Modeling electrical stimulation of mammalian nerve fibers: a mechanistic versus probabilistic approach
}

\author{
Vijay Sadashivaiah, Pierre Sacré, Yun Guan, William S. Anderson, and Sridevi V. Sarma, IEEE Member
}

\begin{abstract}
Electrical neurostimulation is increasingly used over neuropharmacology to treat various diseases. Despite efforts to model the effects of electrical stimulation, its underlying mechanisms remain unclear. This is because current mechanistic models just quantify the effects that the electrical field produces near the fiber and do not capture interactions between stimulus-initiated action potentials (APs) and underlying physiological activity initiated APs. In this study, we aim to quantify and compare these interactions. We construct two computational models of a nerve fiber of varying degrees of complexity (probabilistic versus mechanistic) each receiving two inputs: the underlying physiological activity at one end of the fiber, and the external stimulus applied to the middle of the fiber. We then define reliability, $R$, as the percentage of physiological APs that make it to the other end of the nerve fiber. We apply the two inputs to the fiber at various frequencies and analyze reliability. We find that the probabilistic model captures relay properties for low input frequencies $(<10 \mathrm{~Hz})$ but then differs from the mechanistic model if either input has a larger frequency. This is because the probabilistic model only accounts for only (i) inter signal loss of excitability and (ii) collisions between stimulus-initiated action potentials (APs) and underlying physiological activity initiated APs. This first step towards modeling the interactions in a nerve fiber opens up opportunities towards understanding mechanisms of electrical stimulation therapies.
\end{abstract}

\section{INTRODUCTION}

Electrical stimulation of mammalian nerve fibers has been of prime interest due to its applications in treating various diseases. For example, Stimulation of peripheral and dorsal column fibers is used to alleviate acute and chronic pain [1]-[4]; In most cases, these nerve fibers have ongoing physiological activity that interacts with the external current stimulus. There are three main interactions: (i) collision block due to annihilation of antidromic stimulus initiated APs with orthodromic APs from ongoing physiological activity, (ii) inter signal loss of excitability of fiber by ongoing physiological activity initiated AP due to recent stimulus AP (physiological-stimulus), and vice versa (stimulusphysiological), (iii) intra signal loss of excitability of the fiber by stimulus initiated AP due to recent stimulus AP (stimulus-stimulus) and by ongoing physiological activity initiated AP due to recent ongoing physiological activity AP

V. Sadashivaiah, P. Sacré and S. V. Sarma are with the Institute for Computational Medicine and the Department of Biomedical Engineering, The Johns Hopkins University, Baltimore, MD (vjs@jhu.edu, p.sacre@jhu.edu, sree@jhu.edu).

Y. Guan is with the Department of Anesthesiology/Critical Care Medicine, The Johns Hopkins University School of Medicine, Baltimore, MD(yguan1@jhmi.edu).

W. S. Anderson is with the Institute for Computational Medicine and the Department of Neurosurgery, The Johns Hopkins University School of Medicine, Baltimore, MD (wanders5@jhmi.edu). (physiological-physiological) [5]. In order to better understand and optimally design stimulation therapies for diseases, it is necessary to understand when and how often each of these interactions occur under different stimulation protocols.

There have been computational efforts to study excitability and interactions in a mammalian nerve fiber. Over the past few decades, biophysical conductance based models of nerve fibers have been developed to study their excitability properties [6]-[8] to name a few. These studies characterize the effects of stimulation on the fiber, such as activation threshold and conduction velocity, by modeling different nerve geometries (single cable or double cable), different tissue mediums, and different electrode configurations, but do not study interactions between stimulus generated APs with ongoing physiological activity in the fiber.

In this study, we characterized all three types of interactions between antidromic APs generated by a deterministic periodic external stimulus and orthodromic physiological APs generated by a stochastic Poisson process. Specifically, we constructed two models of varying degrees of complexity (probabilistic and mechanistic) of a single nerve fiber. The probabilistic model is a simple model that captures the probability of a physiological AP reaching the other end of nerve fiber based on length of nerve fiber (l), speed of conduction $(c)$ and refractory periods. The mechanistic model is a detailed model of single cable nerve fiber that captures high resolution action potential interactions based on biophysical principles. In each model, the nerve fiber receives two inputs: the ongoing physiological activity at one end of the fiber and the external stimulus applied to the middle of the fiber (see Figure 1). We then compute reliability, defined as the ratio of number of physiological APs that make it to the other end of the fiber over the total number of physiological APs entering nerve fiber. Reliability depends on stimulus and physiological activity signal parameters including but not limited to frequency, amplitude and pattern (tonic vs bursting vs stochastic). In this study, we vary the frequency of the input signals and analyze reliability under different fiber diameters.

Our results suggest that, the probabilistic model (runtime of the order of seconds) and the mechanistic model (runtime of the order of days) have similar reliability at low input frequencies $(<10 \mathrm{~Hz})$ but quickly diverge at higher frequencies because the probabilistic model captures less interactions. At low input signal frequencies, interactions in the fiber are mainly due to collisions and inter signal loss of excitability, which is what the probabilistic model accounts for. On the other hand, at high frequencies the interactions are mainly 


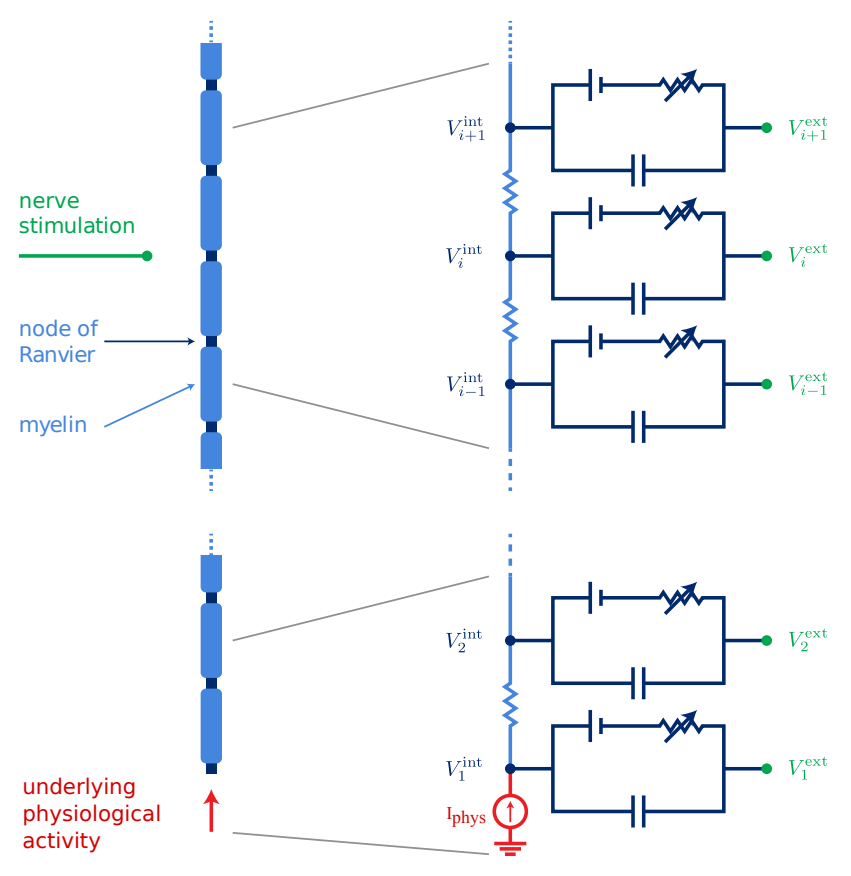

Fig. 1. Mechanistic model of nerve fiber. Our model of extracellular electrical stimulation of myelinated nerve fibers includes the underlying physiological activity as a current source at one end of the nerve fiber.

due to intra signal loss of excitability. Finally, increasing the axon diameter increases reliability for all input frequencies.

This study demonstrates that complex interactions occur between physiological and electrical stimulus along the nerve fibers and it may be useful to characterize these interactions to better understand the mechanisms of action of electrical stimulation used to treat diseases of the nervous system.

\section{MODEL DESCRIPTION}

\section{A. Mechanistic model of a nerve fiber}

Methods used in this section are derived from [5]. Here, we describe our simulation test bed of extracellular electrical stimulation on myelinated nerve fibers with underlying physiological activity (see Figure 1).

\section{B. Probabilistic model of a nerve fiber}

TABLE I

Model Parameters COMPUTED From the Mechanistic MOdel

\begin{tabular}{|l|l|l|l|}
\hline & \multicolumn{3}{|c|}{ Fiber diameter $(\mu \mathrm{m})$} \\
\hline & 6 & 9 & 12 \\
\hline speed of AP conduction $\left(\mathrm{m} \mathrm{s}^{-1}\right)$ & 41.66 & 66.67 & 90.91 \\
\hline phys-stim refractory period $(\mathrm{ms})$ & 9.5 & 7.8 & 7.7 \\
\hline nerve fiber length $(\mathrm{cm})$ & 10 & 10 & 10 \\
\hline simulation time $(\mathrm{s})$ & 30 & 30 & 30 \\
\hline number of realizations (-) & 50 & 50 & 50 \\
\hline
\end{tabular}

In this section, we describe our probabilistic model of nerve fibers. In this model, the nerve fiber is characterized by the geometry (length $(l))$ and speed of conduction $(c)$ (see Table I). Using these properties we compute the probability of different events along the fiber. We assume that:
- The underlying physiological activity follows a poission distribution (rate $=\lambda_{\text {phys }}$ ) and the extracellular stimulus is periodic (frequency $=T_{\text {stim }}$ );

- The interactions are collisions and physiological to stimulus loss of excitability only;

Under these assumptions we compute the reliability as,

$$
\begin{aligned}
R_{t} & =\frac{\text { Number of relayed phsyiological } A P s}{\text { Total number of physiological APs }(T)} \\
& =\frac{n T_{\text {stim }} \lambda_{\text {phys }}-n \operatorname{Pr}(\text { collision })}{n T_{\text {stim }} \lambda_{\text {phys }}}
\end{aligned}
$$

We can derive the $\operatorname{Pr}($ collision $)$ to be,

$$
\begin{aligned}
\operatorname{Pr}(\text { collision }) & =1-\operatorname{Pr}(\text { no collision }) \\
& =e^{\lambda_{\text {phys }} r_{\text {phys }- \text { stim }}}\left(1-e^{\lambda_{\text {phys }}(2 l) / c}\right)
\end{aligned}
$$

where $r_{\text {phys-stim }}$ is the refractory period in nerve fiber due to a recent physiological AP.

\section{Electrical field potential generated by stimulation.}

The extracellular medium may be assumed to be infinite and isotropic with the electrode represented by point sources at the center $x_{j}^{\mathrm{c}}$ of each contact. Therefore, the electrical potential field at time $t$ and position $x$ is given by

$$
\varphi(t, x)=\sum_{j \in \mathcal{C}} \frac{\rho_{\mathrm{m}}}{4 \pi\left\|x-x_{j}^{\mathrm{c}}\right\|_{2}} I_{j}^{\mathrm{stim}}(t),
$$

where $I_{j}^{\text {stim }}$ is the current of point source $j$ and $\rho_{\mathrm{m}}$ is the extracellular medium resistivity. The extracellular potential at node $i$ is given by $V_{i}^{\text {ext }}(t)=\varphi\left(t, x_{i}\right)$, where $x_{i}$ is the position of node $i$.

The stimulation current input $I^{\text {stim }}(t)$ consists of the repetition, at a constant frequency, $f^{\text {stim }}$, of symmetrical biphasic pulses with an amplitude ranging from $1.8 \mathrm{~mA}$ to $2.5 \mathrm{~mA}$ (increasing activation thresholds as fiber diameter increases) and a duration of $350 \mu \mathrm{s}$ [9]. We consider stimulation frequencies ranging from 1 to $50 \mathrm{~Hz}$.

\section{Underlying physiological activity.}

The underlying physiological activity in fibers spans a broad frequency range and exhibits various patterns [10]: regular spike discharge, regular discharge of doublet spikes, bursting patterns, sporadic activity with no regular or predictable firing pattern, etc.

The presence of underlying activity in the nerve fiber is represented by replacing a 'sealed-end' boundary condition by a current source at one end of the nerve fiber. Therefore, the dynamics of the first node becomes

$C_{\mathrm{m}} \dot{V}_{1}+\sum_{k \in \mathcal{K}} I_{1, k}=G_{\mathrm{a}}\left(V_{2}-V_{1}\right)+G_{\mathrm{a}}\left(V_{2}^{\mathrm{ext}}-V_{1}^{\mathrm{ext}}\right)+I^{\mathrm{phys}}(t)$,

where the input $I^{\text {phys }}(t)$ represents the underlying activity.

As a first step, the underlying physiological activity input $I^{\text {phys }}(t)$ is modeled as a Poisson train of square pulses with an amplitude of $4 \mathrm{nA}$ to $7 \mathrm{nA}$ (increasing activation thresholds as fiber diameter increases) and a duration of 
$1 \mathrm{~ms}$. Therefore, the instantaneous firing rate $\lambda^{\text {phys }}$ is assumed constant, ranging from $1 \mathrm{~Hz}$ to $50 \mathrm{~Hz}$, a typical range for motor and sensory firing activity [11], [12].

\section{E. Reliability}

When stimulation is applied to a nerve fiber, it ultimately is interfering with the ongoing physiological activity that travels from one end of the fiber to the other. To succinctly quantify the effects of stimulation on the fiber activity, we wanted to capture how the stimulation influences the physiologically generated APs that make it to the other end of the fiber. To quantify the effects of stimulation on the nerve fiber, we define the following reliability metric:

$$
R^{\text {phys }}\left(\lambda^{\text {phys }}, f^{\text {stim }}\right)=\frac{\# \text { of relayed physiological APs }}{\text { total \# of physiological APs }},
$$

where relayed action potentials are underlying physiological inputs that travel from one end to the other end of the fiber. Metric (7) captures the effect that the stimulation has on the ongoing physiological activity. If $R=1$ then the stimulation has no effect, and if $R=0$, then the stimulation blocks all physiological activity from transmitting to the brain.

\section{RESULTS}

In this section, we show results of our stimulation test beds for a monopolar electrode placed $3.5 \mathrm{~mm}$ from the center of a $\mathrm{L}=10 \mathrm{~cm}$-long nerve fiber. We consider three different diameters of nerve fibers in our simulations $(6 \mu \mathrm{m}$, $9 \mu \mathrm{m}$ and $12 \mu \mathrm{m})$. The results presented in this section are drawn from 50 simulations for each frequency pair (physiological, stimulus) of this model with a stochastic Poisson physiological input. All the mechanistic model simulations were performed on NEURON simulation environment [13]. Analysis of data and probabilistic model simulations were performed on MATLAB, MathWorks.

\section{A. Reliability: Probabilistic vs Mechanistic model}

Figure 2 shows examples of a physiological reliability map for probabilistic and mechanistic model for three different diameters $(6 \mu \mathrm{m}, 9 \mu \mathrm{m}$ and $12 \mu \mathrm{m})$. We see that at low stimulation and physiological frequencies $(1 \mathrm{~Hz}$ to $10 \mathrm{~Hz})$, reliability is almost $1(100 \%)$ and thee is more agreement bwteeen the two models. As the stimulus frequency increases, reliability decreases. Also, the probabilistic model captures relay properties that the mechanistic model captures in significantly less run time and with less computational power. Although the values captured by the probabilistic model is much different comapred to mechanistic model. As the axon diameter increases from $6 \mu \mathrm{m}$ to $12 \mu \mathrm{m}$ the reliability value increases at each frequency pair.

\section{B. Influence of fiber diameter on reliability}

For different fiber diameters, the reliability maps change (see Figure 2). We observe a horizontal shift in the pattern as we go from $6 \mu \mathrm{m}$ to $12 \mu \mathrm{m}$ diameter fibers. Consider a stimulus frequency of $50 \mathrm{~Hz}$ and physiological frequency of (a) Probabilistic model

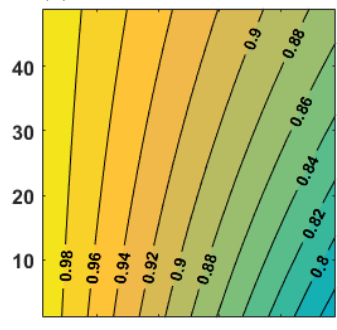

(b)

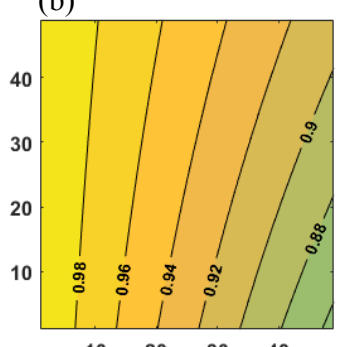

(c)
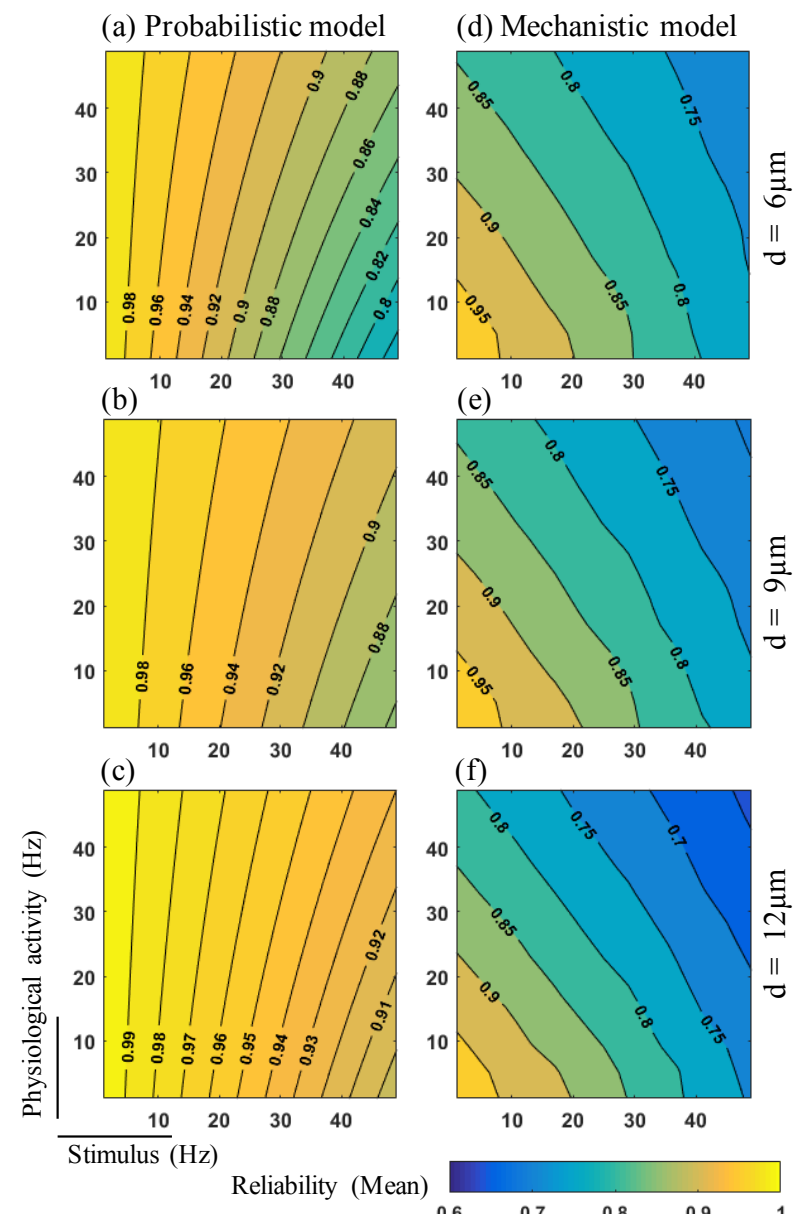

(e)

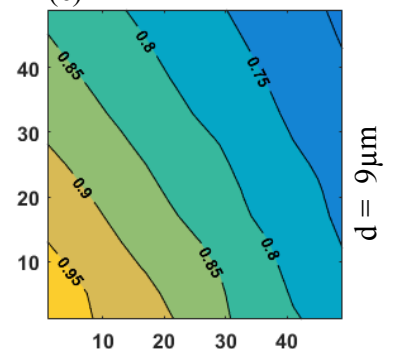

(f)

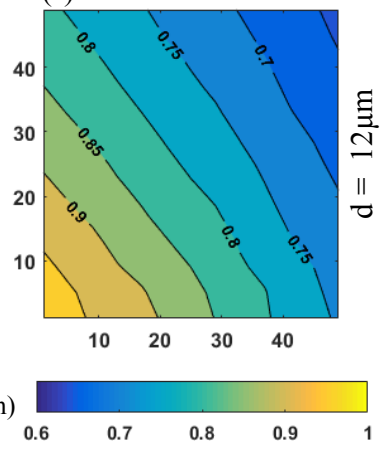

Fig. 2. Reliability maps of probabilistic and mechanistic model for $6 \mu \mathrm{m}$, $9 \mu \mathrm{m}$ and $12 \mu \mathrm{m}$ diameters. Contour map of reliability values for a range $(1 \mathrm{~Hz}$ to $50 \mathrm{~Hz})$ of physiological frequency (Y-axis) and stimulus frequency (X-axis). Color gradient represents the mean of reliability values $(0.6-1)$.

$10 \mathrm{~Hz}$, we see that the reliability values for $12 \mu \mathrm{m}$ is greater than that of $9 \mu \mathrm{m}$ which is greater than $6 \mu \mathrm{m}$. This is because of the low conduction velocity of AP's in small fibers. With an increase in fiber diameter, reliability increases as the interaction count is reduced at higher conduction velocities. Figure 3(b,c) shows the difference between the reliability maps of $6 \mu \mathrm{m}$ and $12 \mu \mathrm{m}$ diameter fibers for probabilistic and mechanistic models.

\section{Discussion}

In this work, we study the interactions between electrical stimulation and physiological activity induced action potentials in a mammalian nerve fiber. We found that reliability depends on stimulus frequency and physiological parameters such as frequency and diameter of the nerve fiber, both affecting the interactions between APs generated by both inputs.

\section{A. Probabilistic versus mechanistic model}

The probabilistic model captures many relay properties that the mechanistic model captures, but with significantly 

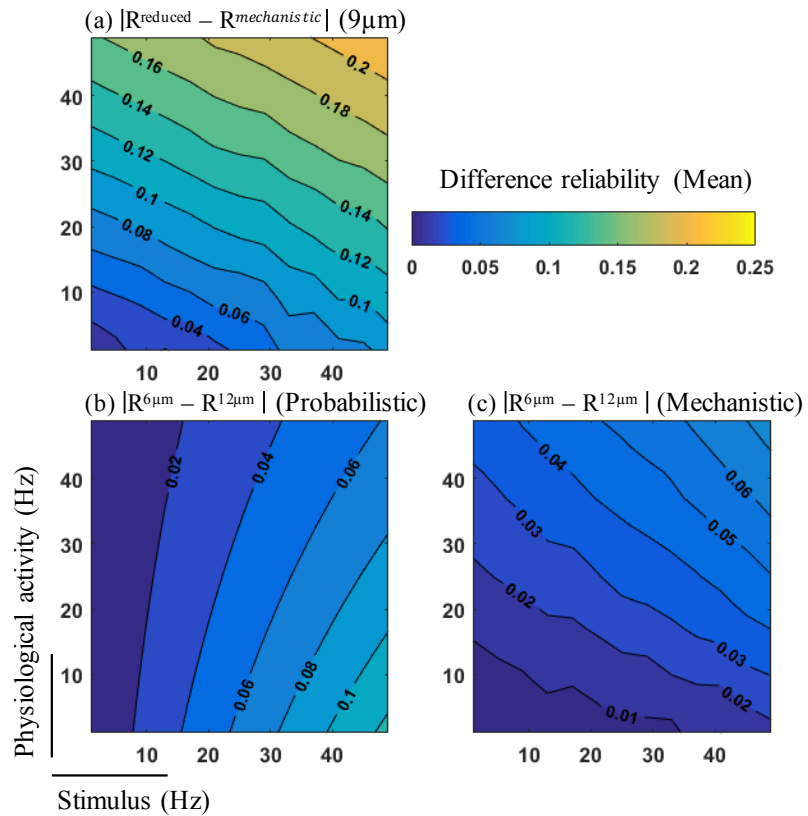

Fig. 3. Influence of diameter on reliability. a. Reliability map of abs(reduced - mechanistic) model for $9 \mu \mathrm{m}$ diameter. b,c. Reliability map of $\operatorname{abs}(6 \mu \mathrm{m}$ vs $12 \mu \mathrm{m})$ diameters for probabilistic and mechanistic models respectively.

less time and less computation power. This is because the mechanistic model involves solving multi-dimensional differential equations at each fiber node to compute signal transmission. In contrast, the probabilistic model uses the fiber length, speed of conduction, and refractory periods to compute reliability statistics.

As expected, we found that the reliability is high at low physiological and stimulus frequencies, because fewer interactions between simulation-evoked activity and underlying physiological activity occur. As the frequencies of stimulus and physiological inputs increase, reliability decreases. This is due to the increase in number of interactions. The differences between the probabilistic and mechanistic model at high frequency stimulation suggest that fiber behavior cannot be quantified by the collision - loss of excitability model, but involves more interactions.

The poisson process sometimes generates interval that is shorter than its pulse width (thus, a spike is generated by doublet signal pulses), and that may have an influence on the reliablity in the probablistic model. In the future, we plan (i) to consider more complex physiological inputs than Poisson, such as doublets or bursts, (ii) to add more interactions into the existing probabilistic model.

\section{B. Influence of fiber diameter on reliability.}

We see from Figure 3(b,c), that increasing the diameter of the nerve fiber from $6 \mu \mathrm{m}$ to $12 \mu \mathrm{m}$ shifts the reliability map to the right. This is expected, since the conduction speeds are higher in a bigger fiber [14], and therefore requires a higher frequency stimulus to achieve the relay properties observed for lower stimulus frequencies in smaller fibers.

\section{ACKNOWLEDGMENT}

Work supported by NIH R01 AT009401 to S.V.S, Y.G. , and W.S.A. We would like to thank Dr. M. Caterina, Neurosurgery Pain Research Institute, The Johns Hopkins University School of Medicine, for valuable and insightful discussions.

\section{REFERENCES}

[1] C. N. Shealy, J. T. Mortimer, and J. B. Reswick, "Electrical inhibition of pain by stimulation of the dorsal columns: preliminary clinical report." Anesthesia \& Analgesia, vol. 46, no. 4, pp. 489-491, 1967.

[2] P. D. Wall and W. H. Sweet, "Temporary abolition of pain in man," Science, vol. 155, no. 3758, pp. 108-109, 1967.

[3] D. A. Stidd, A. Wuollet, K. Bowden, T. Price, A. Patwardhan, S. Barker, M. E. Weinand, J. Annabi, and E. Annabi, "Peripheral nerve stimulation for trigeminal neuropathic pain," Pain Physician, vol. 15, no. 1, p. 27, 2012.

[4] R. Shechter, F. Yang, Q. Xu, Y.-K. Cheong, S.-Q. He, A. Sdrulla, A. F. Carteret, P. W. Wacnik, X. Dong, R. A. Meyer et al., "Conventional and kilohertz-frequency spinal cord stimulation produces intensity-and frequency-dependent inhibition of mechanical hypersensitivity in a rat model of neuropathic pain," Anesthesiology, vol. 119, no. 2, pp. 422 432, 2013.

[5] P. Sacré, S. V. Sarma, Y. Guan, and W. S. Anderson, "Electrical neurostimulation for chronic pain: on selective relay of sensory neural activities in myelinated nerve fibers," in Proceeding of the 37th Annual International Conference of the IEEE Engineering in Medicine and Biology Society (EMBC), 2015, pp. 4705-4708.

[6] J. P. Reilly, "Peripheral nerve stimulation by induced electric currents: exposure to time-varying magnetic fields," Medical and Biological Engineering and Computing, vol. 27, no. 2, pp. 101-110, 1989.

[7] W. A. Wesselink, J. Holsheimer, and H. B. K. Boom, "A model of the electrical behaviour of myelinated sensory nerve fibres based on human data," Medical \& Biological Engineering \& Computing, vol. 37, no. 2, pp. 228-235, 1999.

[8] C. C. McIntyre, A. G. Richardson, and W. M. Grill, "Modeling the excitability of mammalian nerve fibers: influence of afterpotentials on the recovery cycle," Journal of Neurophysiology, vol. 87, no. 2, pp. 995-1006, 2002.

[9] J. T. Mortimer and N. Bhadra, "Peripheral nerve and muscle stimulation," in Neuroprosthetics: Theory and Practice, K. W. Horch and G. S. Dhillon, Eds. World Scientific, 2004, pp. 638-682.

[10] K. C. Kajander and G. J. Bennett, "Onset of a painful peripheral neuropathy in rat: a partial and differential deafferentation and spontaneous discharge in A beta and A delta primary afferent neurons,' Journal of Neurophysiology, vol. 68, no. 3, pp. 734-744, 1992.

[11] B. Katz, "Action potentials from a sensory nerve ending," The Journal of Physiology, vol. 111, no. 3-4, p. 248, 1950.

[12] C. J. De Luca, R. S. LeFever, M. P. McCue, and A. P. Xenakis, "Behaviour of human motor units in different muscles during linearly varying contractions," The Journal of Physiology, vol. 329, no. 1, pp. 113-128, 1982.

[13] M. L. Hines and N. T. Carnevale, "The NEURON simulation environment," Neural Computation, vol. 9, no. 6, pp. 1179-1209, 1997.

[14] J. B. Hursh, "Conduction velocity and diameter of nerve fibers," American Journal of Physiology, vol. 127, no. 1, pp. 131-139, May 1939. 